Provided for non-commercial research and education use. Not for reproduction, distribution or commercial use.

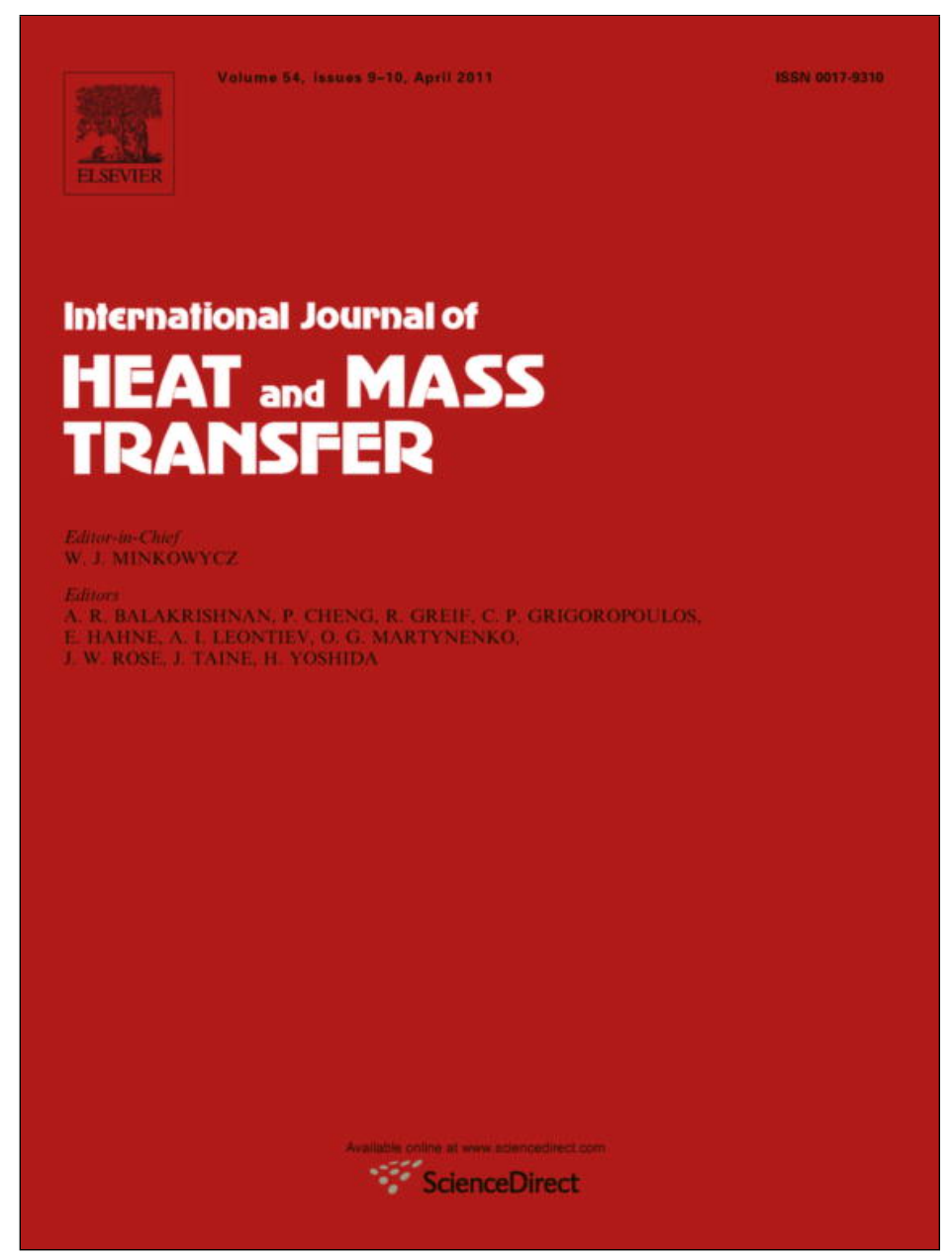

This article appeared in a journal published by Elsevier. The attached copy is furnished to the author for internal non-commercial research and education use, including for instruction at the authors institution and sharing with colleagues.

Other uses, including reproduction and distribution, or selling or licensing copies, or posting to personal, institutional or third party websites are prohibited.

In most cases authors are permitted to post their version of the article (e.g. in Word or Tex form) to their personal website or institutional repository. Authors requiring further information regarding Elsevier's archiving and manuscript policies are encouraged to visit:

http://www.elsevier.com/copyright 


\title{
A Monte Carlo simulation for phonon transport within silicon structures at nanoscales with heat generation
}

\author{
Basil T. Wong ${ }^{\mathrm{a}, *}$, Mathieu Francoeur ${ }^{\mathrm{b}}$, M. Pinar Mengüç, ${ }^{\mathrm{c}, \mathrm{d}}$ \\ ${ }^{a}$ Swinburne University of Technology, Sarawak Campus, School of Engineering, Computing and Science, Room E203, Block E, Jalan Simpang Tiga, 93350 Kuching, Sarawak, Malaysia \\ ${ }^{\mathrm{b}}$ Department of Mechanical Engineering, University of Utah, 50 S. Central Campus Dr., 2126 MEB, Salt Lake City, UT 84112, USA \\ ${ }^{\mathrm{c}}$ Center for Energy, Environment and Economy, Özyeğin University, Altunizade 34662, Istanbul, Turkey \\ ${ }^{\mathrm{d}}$ Department of Mechanical Engineering, University of Kentucky, 151 RGAN Building, Lexington, KY 40506, USA
}

\section{A R T I C L E I N F O}

\section{Article history:}

Received 9 April 2010

Received in revised form 10 December 2010

Accepted 10 December 2010

Available online 14 February 2011

\section{Keywords:}

Monte Carlo

Phonon transport

Silicon thin film

Heat generation

Ballistic transport

Near-field thermal radiation

The Fourier law

Heat diffusion equation

\begin{abstract}
A B S T R A C T
Nanoscale phonon transport within silicon structures subjected to internal heat generation was explored. A Monte Carlo simulation was used. The simulation procedures differed from the current existing methods in which phonons below a predefined "reference temperature" were not accounted to reduce memory storage and computational resources. Results indicated that the heat diffusion equation significantly underestimates temperature distribution at nanoscales in the presence of an external heat source. Discussions on temperature distribution inside silicon thin film when heated by a pulsed laser, an electron beam or due to near-field thermal radiation effects were also provided.
\end{abstract}

(c) 2011 Elsevier Ltd. All rights reserved.

\section{Introduction}

At macroscales, the rate of heat conducted through an area is proportional to the thermal conductivity and the temperature gradient, following the Fourier law [1-4]. The basic premise of the law is that the characteristic length of the object must be greater than the mean free path of the heat carriers. Using this phenomenological law to represent heat fluxes on all the surfaces of an object, the heat diffusion equation can be derived by simply summing all these fluxes and equating them to the rate of change of its internal energy. Even though the Fourier law is generally applicable at the macroscales, it is commonly used at the microscales to represent thermal heat flux of electrons and phonons [2,5-9]. For example, in the ultra-fast heating of metallic films where highly non-equilibrium phenomena is observed, the two-temperature model (TTM) is used to simulate thermal conduction of electrons and phonons [9-11]. The TTM basically consists of two coupled diffusion equations where heat fluxes of electrons and phonons are calculated separately. This formulation is also employed in the electron-phonon hydrodynamic equation (EPHDEs)

\footnotetext{
* Corresponding author.

E-mail addresses: twong@swinburne.edu.my (B.T. Wong), mfrancoeur@mech. utah.edu (M. Francoeur), menguc@engr.uky.edu, pinar.menguc@ozyegin.edu.tr (M. Pinar Mengüç).
}

to express the thermal flux of electrons and the heat flux of phonons as a function of corresponding thermal conductivity and temperature gradient $[2,9,12]$. However, when the characteristic length of an object is smaller than the mean free path, which is commonly observed at nanoscales, heat conduction no longer obeys the Fourier law, mainly due to the impact of ballistic propagation by the heat carriers. At such scales, thermal conductivity and temperature gradient are reduced while discontinuity in the temperature distribution near the boundary exists [13-20]. Therefore, either the general Boltzmann transport equation (BTE) or the phonon radiative transport equation (PRTE) is required to correctly model the phonon transport $[1,2,21-26]$. The energy equations for electrons and phonons in the TTM and the EPDHEs where the diffusion approximation is assumed are to be substituted by the corresponding BTE in the intensity form in order to account for the ballistic behaviors of heat carriers. This can be done only when the average mean free path of electrons/phonons exceeds the physical length of the system. Typical electron and phonon mean free paths range from 1 to $500 \mathrm{~nm}$ depending on the wavelength and energy $[1,2,23,27]$. An object with characteristic dimensions less than the mean free paths would generally exhibit (semi-) ballistic behavior.

Among analytical and numerical methods available to solve the BTE $[1,2,4,23,26,28]$, Monte Carlo (MC) simulations are proven to 


\begin{tabular}{|c|c|c|c|}
\hline \multicolumn{4}{|c|}{ Nomenclature } \\
\hline$c_{v}$ & speed of light in vacuum [m/s] & Symbols & \\
\hline$D$ & density of states $\left[\mathrm{m}^{-3}-\mathrm{s}\right]$ & $\mu$ & direction cosine $[-]$ \\
\hline E & energy $[\mathrm{eV}]$ & $\tau$ & relaxation time $[\mathrm{s}]$ \\
\hline$f$ & particle distribution function [-] & $\Theta$ & mean energy of a Planck oscillator, $J$ \\
\hline$\dot{g}^{\prime \prime \prime}$ & power density/volumetric heat generation $\left[\mathrm{W} / \mathrm{m}^{3}\right]$ & $\beta$ & parallel component of the wavevector $[\mathrm{rad} / \mathrm{m}]$ \\
\hline$g_{s l}$ & $\begin{array}{l}\text { Weyl component of the dyadic Green's function be- } \\
\text { tween layers } s \text { and } l[\mathrm{~m}]\end{array}$ & $\gamma$ & $\begin{array}{l}\text { perpendicular component of the wavevector }\left(=\gamma^{\prime}+i \gamma^{\prime \prime}\right) \\
{[\mathrm{rad} / \mathrm{m}]}\end{array}$ \\
\hline$i$ & complex constant, $(-1)^{1 / 2}[-]$ & $\varepsilon_{r}$ & dielectric function $\left(=\varepsilon_{r}^{\prime}+i \varepsilon_{r}^{\prime \prime}\right)$ \\
\hline$k$ & wavenumber $\left[\mathrm{m}^{-1}\right]$ & $\rho, \theta, z$ & polar coordinate system \\
\hline$k_{T}$ & thermal conductivity [W/m-K] & & angular frequency [ $\mathrm{rad} \mathrm{s}^{-1}$ ] \\
\hline$k_{B}$ & Boltzmann's constant $[\mathrm{eV} / \mathrm{K}]$ & \multicolumn{2}{|c|}{ Subscripts } \\
\hline$N$ & number of phonons or ensembles [-] & $*$ & complex conjugate \\
\hline$N_{b}$ & number of division in phonon frequency domain $[-]$ & 0 & equilibrium \\
\hline$p$ & polarization branch [-] & $E$ & electric \\
\hline$P$ & probability $[-]$ & en & ensemble \\
\hline$R$ & cumulative probability distribution function $[-]$ & gen & heat generation \\
\hline Ran & a random number $[-]$ & $H$ & magnetic \\
\hline$S$ & distance of interaction [m] & ini & initial \\
\hline$T$ & temperature [K] & $L A$ & longitudinal acoustic \\
\hline$t$ & time $[\mathrm{s}]$ & $m$ & medium \\
\hline$v_{g}$ & group velocity $[\mathrm{m} / \mathrm{s}]$ & ph & phonon \\
\hline$X$ & width of the geometry [m] & ref & reference \\
\hline Y & length of the geometry [m] & $T A$ & transverse acoustic \\
\hline$Z$ & depth (or thickness) of the geometry [m] & $\omega$ & monochromatic \\
\hline
\end{tabular}

be the most flexible and accurate, yet they can be slow and expensive in terms of computational resources depending on the levels of physics included in the simulation. Many researchers have used MC simulations for phonon transport at nanoscales because of its flexibility in accounting complicated geometries and the correct phonon dispersion relation and different polarization branches $[14,16-20,25,29-31]$. While the simulation has been successfully used for predicting thermal conductivities of nanostructures such as nanowires and nanofilms [14,18-20,31-34], there is plenty of room for improvement in the algorithm, especially for treating the phonon-phonon scattering mechanisms. On the other hand, the effect of external heat generation on phonon transport near ballistic limit has never been studied, which is the focus of this work.

To explore phonon transport at nanoscales, here we introduce a new MC simulation procedure to solve phonon transport within a 3D-rectangular geometry, as depicted in Fig. 1. The model is a rectangular system $(X \times Y \times Z)$ where constant temperature is applied at both ends along the $Z$-dimension while the other surfaces are assumed insulated. Depending on the dimensions of $(X \times Y)$, the geometry can be considered as a $Z \mathrm{~nm}$ thin film with infinite $X$ and $Y$, a nanorod with comparable magnitudes of $X, Y$, and $Z$, or a nanowire where $Z>>X$ and $Y$. The 2D top view of the geometry is also provided in Fig. 1 to illustrate phonon activities during the transport process. Phonons are emitted from the constant temperature boundaries while additional phonons are generated within the medium/material as a result of external heating. We do not prescribe the type of external heating in the simulations because this is irrelevant as long as the heating process directly produces energetic phonons corresponding to the amount of the volumetric heat generation specified. The constant temperature boundaries are assumed to be perfect absorbers. In the simulations, the insulated surfaces along the $Z$-dimension can be of specular or diffusive type. As the names implied, a specularly insulated surface acts like a mirror while a diffusive insulated surface reflects phonons diffusively upon encountering. Reflection in the latter can be regarded as the effect of surface roughness.
In the following sections, a modified MC simulation procedure used in this formulation is first explained in detail including all the phonon scattering properties and algorithms for determining scattering processes of phonons. These procedures are general and can be adapted for simulating phonon transport in any material; in this work we explicitly use the properties of silicon. Simulations for other materials will be carried out in another study. The MC simulations are verified against known analytical solutions at the ballistic and diffusive limits. Next, the impacts of ballistic phonon transport on temperature distribution are studied for different heat generation distributions. Finally, we provide discussions and potential future works on the applications of the MC results coupled with near-field thermal radiation, laser heating, or electron-beam heating.

\section{A modified Monte Carlo simulation in phonon transport}

A typical MC simulation strategy for phonon transport is relatively straight forward: we initialize, launch, and trace phonon ensembles in terms of temperature, frequency, polarization, and positions. Local temperature distribution varies depending on the positions of these ensembles. A general flowchart of the MC simulation is shown in Fig. 2. The simulation starts by initializing all the phonon ensembles (including those to be launched from the constant-temperature boundary and within the material due to heat generation at each $\Delta t$ ). These ensembles are moved ballistically from one position to another within the time interval of $\Delta t$, assuming that ensemble properties remain unaltered. Ensembles that hit a constant-temperature boundary are recorded in terms of energy for heat flux calculation and then deleted. Those that encounter an insulated surface are assumed to be reflected specularly or diffusively. Otherwise, ensembles reside at the corresponding locations after the ballistic movement. Once the propagation phase is complete, local temperature distribution is calculated based on the positions of the ensembles. It is important to notice that local phonon distribution function after the propagation phase is different from the equilibrium distribution (i.e., the Bose-Einstein distribu- 
tion). Only the local distribution of total phonon energy is known. Although a medium can still be in a non-equilibrium state, it is possible to calculate "pseudo-temperature" distribution assuming that equilibrium exists [14]. Discussions on how the "pseudotemperature" is calculated is provided later. When the local (pseudo-) temperature distribution is known, the corresponding phonon scattering properties can be evaluated properly. The probabilities of scattering for the ensembles are calculated based on the scattering properties of phonons at the "pseudo-temperature." If an ensemble is scattered, its frequency and polarization are reset based on the equilibrium distributions. Details involving all the above steps are given in the following sections.

Our MC simulation for phonon transport differs from those in the literature $[14,16-17,32]$ in the ways of handling the phonon initialization and scattering procedures. In our preliminary calculations we have observed that initializing all the phonons for a given temperature in the simulation was utterly time-consuming, and it required significant amount of computational resources to store histories of phonons, even if a scaling factor was used. In addition, a large amount of phonon ensembles was required to minimize statistical noises in the temperature distribution. To overcome this drawback, we set a "reference temperature" above which phonons are initialized over the entire frequency spectrum. In other words, only phonons in addition to those of the "reference temperature" are simulated and temperature is never allowed to fall below the reference value. The advantage of this method is that computational resources and statistical noises can be minimized. The "reference temperature" is usually set to the lowest temperature in the medium. It is possible that phonons below the reference temperature, which are not considered in the simulation, are scattered through $N$ - or $U$ - processes. However, these events happen over the entire domain and every possible direction, and therefore yield no effect to the overall heat transfer since they cancel out each other. We compared MC simulations with and without the prescribed reference temperature, and both demonstrated statistically similar results. Extending this type of MC method to other applications/problems should require careful consideration since temperature should not fall below the reference temperature throughout the entire domain.

In order to utilize this adjustment, the remaining MC procedures should be changed accordingly, which are discussed below.

\subsection{Phonon descriptions - dispersion relation, density of states, and group velocity}

For the sake of simplicity, dispersion relation of silicon in any given direction is assumed to be identical to that in the (001) direction. The data points of the dispersion relation for silicon are provided by Brockhouse [35]. Quadratic expressions are used to fit these dispersion data. Using the fitted expressions, calculating the angular frequency with known wave vector or vice versa can be achieved easily during a MC simulation. The dispersion relation used in this work is shown in Fig. 3, and the quadratic expressions for the LA- and TA-dispersion relations are obtained as:

$\omega=9280 k-2.234 \times 10^{-7} k^{2}(L A)$,

$\omega=5240 k-2.278 \times 10^{-7} k^{2}(T A)$.

When the dispersion relation is known, the phonon density of states for a given polarization branch, $D(\omega, p)$, is calculated as:

$D(\omega, p)=\frac{k^{2}}{2 \pi^{2} v_{g}(\omega, p)}$,

and the group velocity of the phonon, $v_{g}(\omega, p)$, is given as: $v_{g}(\omega, p)=\frac{\partial \omega}{\partial k}$.

\subsection{Determining properties of phonon ensembles - quantity, frequency, velocity, and polarization}

To start a MC simulation, the required number of statistical ensembles needs to be specified and initialized. This is done by first calculating the total actual number of phonons present in the medium. Since a "reference temperature" is set in the simulation, only phonons that are created beyond the reference are initialized. Therefore, the initial number of phonons available for carrying excess heat in the medium above the reference is calculated as:

$N_{p h, i n i}=X Y Z \sum_{p} \sum_{i}\left[f_{0}\left(\omega_{i}, T_{i n i}, p\right)-f_{0}\left(\omega_{i}, T_{r e f}, p\right)\right] D\left(p, \omega_{i}\right) \Delta \omega_{i}$,

where the index $p$ includes two transverse acoustic $(T A)$ and one longitudinal acoustic $(L A)$ polarization branches of phonons. Here, the equilibrium distribution function (i.e. $f_{0}$ ) corresponds to the Bose-Einstein distribution, given as:

$f_{0}(\omega, T)=\frac{1}{\exp \left(h \omega / k_{B} T\right)-1}$.

Since it is impossible to track all these phonons individually, a scaling factor, $W_{\text {scaling, }}$ is used to represent the actual number of phonons that each statistical ensemble carries:

$W_{\text {scaling }}=\frac{N_{\text {ph,ini }}}{N_{\text {en }, m}}$

where $N_{e n, m}$ is the initial number of statistical ensembles used to represent the total actual number of phonons present in the medium. The "reference temperature" is set to be the initial temperature meaning that $N_{\text {ph,ini }}^{\text {actal }}=0$ according to Eq. (5) and that the initial number of statistical ensembles in the medium is null. Therefore, we use the temperature at the isothermal boundary to obtain another scaling factor. At each time step, $\Delta t$, the excess number of phonons emitted from a boundary with constant temperature of $T_{R}$ or $T_{L}$ in reference to $T_{\text {ref }}$ at each time step is computed as:

$N_{p h, R}=X Y \Delta t \sum_{p} \sum_{i=1}^{N_{b}}\left[f_{0}\left(\omega_{i}, T_{R}\right)-f_{0}\left(\omega_{i}, T_{r e f}\right)\right]\left[\vec{v}_{g}\left(\omega_{i}\right) \cdot \hat{n}\right] D\left(\omega_{i}, p\right) \Delta \omega_{i}$,

where $\left(\vec{v}_{\omega, g} \cdot \hat{n}\right)$ is the phonon group velocity normal to the boundary. Thus, the scaling factor becomes:

$W_{\text {scaling }}=\frac{N_{p h, R}}{N_{e n, R}}$.

In Eq. (9), $N_{p h, R}$ is to be replaced with $N_{p h, L}$ to calculate the scaling factor, if the latter is larger than the former. We implemented this in our algorithm although one can choose the reverse approach to obtain the scaling factor. Once the scaling factor is determined, it remains identical throughout the entire simulation for consistency. Using the convention given, the number of statistical ensembles to be launched from the $T_{R}$-boundary is simply $N_{e n, R}$ while from the $T_{L}$-boundary is

$N_{e n, L}=\frac{N_{p h, L}}{W_{\text {scaling }}}$.

The next step is to determine the frequency of a phonon ensemble launched from the $T_{R}$-boundary. This is done by first constructing the CPDF of the number of phonons over the frequency spectrum as: 
$R_{i}=\sum_{j=1}^{i} N_{j} / \sum_{j=1}^{N_{b}} N_{j}$

where

$N_{j}=\Delta \omega_{j}\left\{\left[f_{0}\left(\omega_{j}, T_{R}\right)-f_{0}\left(\omega_{j}, T_{r e f}\right)\right] D\left(\omega_{j}, L A\right)+2 D\left(\omega_{j}, T A\right)\right\}$.

In Eq. (11), the frequency spectrum is divided into $N_{b}$ intervals. Then a random number, denoted as $\operatorname{Ran}_{\omega}$, is drawn such that:

$R_{i-1}<\operatorname{Ran}_{\omega}<R_{i}$,

and the actual frequency of the statistical ensemble is calculated as:

$\omega=\omega_{i}+\left(2 \operatorname{Ran}_{\omega}-1\right) \frac{\Delta \omega_{i}}{2}$.

Similarly, equations (11)-(14) are used to determine the frequency of a phonon ensemble originated from the $T_{L}$-boundary with the exception that $T_{R}$ is replaced with $T_{L}$.

When the frequency of the ensemble is known, we can then determine its polarization by computing the ratio of the LA-phonons to the total number of phonons in all polarization branches in the $\omega$ interval, which is given as:

$P_{L A, i}=\frac{\left[f_{0}\left(\omega_{i}, T_{R}\right)-f_{0}\left(\omega_{i}, T_{r e f}\right)\right] D\left(\omega_{i}, L A\right)}{\left[f_{0}\left(\omega_{i}, T_{R}\right)-f_{0}\left(\omega_{i}, T_{r e f}\right)\right]\left[D\left(\omega_{i}, L A\right)+2 D\left(\omega_{i}, T A\right)\right]}$.

A random number, $\operatorname{Ran}_{P}$, is then drawn to compare with $P_{L A, i}$. If $\operatorname{Ran}_{P}$ is less than $P_{L A, i}$, then the ensemble belongs to the $L A$ polarization branch; otherwise, it belongs to the $T A$ polarization branch. The same procedures are applicable for the $T_{L}$-boundary. With the frequency and polarization branch known, the group velocity of the ensemble can be determined easily using Eqs. (1), (2), and (4). Additional phonon ensembles are launched within the medium as a result of the external heat generation; these discussions are provided in Section 2.8 .

\subsection{Sampling initial launching and scattering angles}

The launching and scattering angles of phonons are assumed to be isotropic, i.e., uniform in all directions. Sampling of the initial launching directions of ensembles originated from an isothermal boundary is slightly different from those launched within the medium. At the isothermal boundary, assuming that the emission is isotropic and diffuse, the initial launching directions are sampled from the hemispherical solid angle where the total emission angle is expressed as:

$\int_{0}^{2 \pi} \int_{0}^{\pi / 2} \cos \theta \sin \theta d \theta d \phi=\pi$

and the $\cos \theta$ accounts for surface area of emission at different $\theta$. Building the CPDF of Eq. (16) in terms of $\theta$ yields:

$R(\theta)=2 \int_{0}^{\theta} \cos \theta^{\prime} \sin \theta^{\prime} d \theta^{\prime}=\sin ^{2} \theta$.

Therefore, by replacing $R(\theta)$ with a random number, $\operatorname{Ran}_{\theta}$, the polar angle of emission from the boundary is sampled as:

$\theta=\sin ^{-1} \sqrt{\operatorname{Ran}_{\theta}}$,

and the azimuthal/zenith angle is sampled as:

$\phi=2 \pi \operatorname{Ran}_{\phi}$.

For ensembles that are launched within the medium, the polar angle is sampled from the total solid angle, which is given as:

$\int_{0}^{2 \pi} \int_{0}^{\pi} \sin \theta d \theta d \phi=4 \pi$.

The CPDF for sampling $\theta$ is then derived as:
$R(\theta)=\frac{1}{2} \int_{0}^{\theta} \sin \theta d \theta=\frac{1}{2}(1-\cos \theta)$,

and

$\theta=\cos ^{-1}\left(1-2 \operatorname{Ran}_{\theta}\right)$.

The zenith angle remains identical as given by Eq. (19). For ensemble scattering, the direction of propagation is also reset following Eqs. (19) and (22).

\subsection{3-D tracking/tracing algorithms}

In the case of anisotropic scattering, a fixed coordinate frame and a moving one are needed to track the ensembles; the scattering angles are always drawn with respect to the moving coordinate frame. However, when one assumes isotropic scattering, only a fixed coordinate frame is required as scattering is equally distributed in all directions. Direction cosines and distances between interaction points are crucial for calculating positions of the ensembles within the medium. Knowing the coordinates of an ensemble at its previous position $\left(x_{\text {old }}, y_{\text {old }}, z_{\text {old }}\right)$, the scattered direction cosines $\left(\mu_{x}^{\prime}, \mu_{y}^{\prime}, \mu_{z}^{\prime}\right)$, and the distance of interaction $(S=v g \Delta t)$, the new coordinates of the ensemble $\left(x_{\text {new }}, y_{\text {new }}, z_{\text {new }}\right)$ are obtained from the following relations:

$x_{\text {new }}=x_{\text {old }}+\mu_{x}^{\prime} S$,

$y_{\text {new }}=y_{\text {old }}+\mu_{y}^{\prime} S$,

$z_{\text {new }}=z_{\text {old }}+\mu_{z}^{\prime} S$

The direction cosines of the ensemble with $\theta$ and $\phi$ known are expressed as:

$\mu_{x}^{\prime}=\cos \phi \sin \theta$,

$\mu_{y}^{\prime}=\sin \phi \sin \theta$,

$\mu^{\prime} z=\cos \theta$

\section{5. "Pseudo-temperature" calculation}

Once the statistical ensembles of phonons start to propagate and interchange between small control volumes (or computational elements) within the entire computation domain, the resultant local phonon distribution loses its thermodynamic equilibrium. In order to calculate the local temperature, however, it is necessary to assume that the total energy carried by ensembles of phonons in a local computational element is equal to the total phonon energy computed using the Bose-Einstein distribution for the same volume. The temperature obtained under such condition is called as a "pseudo-temperature," denoted as $T_{\text {pseudo }}$. Therefore, the following equation needs to be solved for the $T_{\text {pseudo }}$ at each time step:

$\sum_{p} \sum_{i=0}^{N_{b}} h \omega_{i}\left[f_{0}\left(\omega_{i}, T_{\text {pseudo }}\right)-f_{0}\left(\omega_{i}, T_{\text {ref }}\right)\right] D_{i}\left(\omega_{i}, p\right) \Delta \omega_{i}=\frac{E(x, y, z)}{\Delta x \Delta y \Delta z}$,

which varies locally. $E(x, y, z)$ is the total energy carried by phonon ensembles within a computational element with $(\Delta x \Delta y \Delta z)$ volume. Eq. (29) can be solved using any numerical method prior the actual MC simulation to construct a table containing a list of the total energy in the computational element with corresponding $T_{\text {pseudo. }}$. Computations can be minimized during the simulation by accessing the table to draw the correct $T_{\text {pseudo }}$ once the total energy is calculated. 


\subsection{Phonon scattering treatment in MC simulation}

Scattering of phonons consists of two types, following either a Normal $(N)$ or an Umklapp $(U)$ process $[1,4,23]$. Both processes tend to restore equilibrium; however, only $U$-processes resist heat conduction. In this work, only three-phonon scattering is accounted during MC simulation although it is probable that fourphonon scattering and beyond may occur at high temperatures, which will be left to future studies. In the three-phonon scattering processes, two phonons can be combined to yield one phonon or a phonon can be decomposed into two separate phonons. Both $\mathrm{N}$ and $U$-processes follow energy conservation as:

$h \omega_{1}+h \omega_{2} \leftrightarrow h \omega_{3}$.

The indices 1, 2, and 3 indicate three different phonons, respectively, and the process given in Eq. (30) is reversible. In addition, phonon scattering follows momentum conservation. For $N$-process, it is given as:

$k_{1}+k_{2} \leftrightarrow k_{3}$.

The $\boldsymbol{k}$ 's are the wave vectors of phonons. For $U$-process, it follows that:

$k_{1}+k_{2} \leftrightarrow k_{3}+G$

where $\boldsymbol{G}$ is the lattice reciprocal vector. Details and physics involved in phonon scattering will not be further discussed here because they are covered extensively elsewhere [1,4,16-17,23].

In the current MC simulation, the parameter involved to account for the phonon scattering is the total relaxation time (i.e., $\left.\tau_{N U}\right)$, which includes both $N$ - and $U$-processes. According to the Mathiessen rule, it is given as $[1,23]$ :

$\frac{1}{\tau_{N U}}=\frac{1}{\tau_{N}}+\frac{1}{\tau_{U}}$.

The CPDF of phonon scattering between $t$ and $t+\Delta t$ is written as:

$R_{\text {scat }}=1-\exp \left(\frac{-\Delta t}{\tau_{N U}}\right)$.

In order to determine if a phonon ensemble is scattered after a time step of $\Delta t$, a random number $\operatorname{Ran}_{\text {scat }}$ is drawn and compared to $R_{\text {scat }}$. If $\operatorname{Ran}_{\text {scat }}$ is less than $R_{\text {scat }}$, the ensemble is scattered. Hence ensemble frequency, polarization, and direction are reset. Relaxation times are typically temperature and frequency dependent; therefore, these quantities are to be calculated at each time step. For silicon, these properties are well-documented. Here, we use the relaxation times expressions proposed by Holland [36] for three-phonon scattering and employed by many researchers in MC simulations [14,16-17] since these expressions produced good fit for the thermal conductivity of silicon in different temperature range. The inverse relaxation times for $N$ - and $U$-processes are given as follows:

LA-phonons and N\&U-processes $\rightarrow \tau_{L A, N U}^{-1}=B_{L} \omega^{2} T^{3}$,

TA-phonons and N-process $\rightarrow \tau_{\text {TA,N }}^{-1}= \begin{cases}B_{T} \omega T^{4} & \forall \omega<\omega_{1 / 2}, \\ 0 & \forall \omega \geqslant \omega_{1 / 2},\end{cases}$

TA-phonons and U- process $\rightarrow \tau_{T A, U}^{-1}=\left\{\begin{array}{ccc}0 & \forall & \omega<\omega_{1 / 2}, \\ \frac{B_{T U} \omega^{2}}{\sinh \left(h \omega / k_{B} T\right)} & \forall & \omega \geqslant \omega_{1 / 2},\end{array}\right.$

where $B_{L}, B_{T}$, and $B_{T U}$ are constants to be determined using bulk thermal conductivity data, and $\omega_{1 / 2}$ is the frequency corresponding to $k / k_{\max }=0.5$ based on the TA-dispersion curve. The values of these constants will be given later.
During the process of phonon scattering, the frequency/energy of each scattered phonon ensemble is reset while additional energy may be added or removed. Thus it is crucial to include an additional step to counteract this imbalance of energy within a control volume. A destruction/creation scheme for phonons can be implemented to prevent any excess energy gain or loss, and the added/ deleted phonons are to be drawn from the equilibrium phonon distribution [14]. However, as discussed by Lacroix et al. [16], the rates of phonon creation and destruction are not equal if scattering processes in the MC simulation are implemented in this way. They suggested modifying the CPDF of sampling the scattered phonon frequency such that phonons with higher scattering rates would have higher probabilities of being drawn at the equilibrium. To do so, Eq. (11) is modified accordingly:

$R_{i}=\sum_{j=1}^{i} N_{j} R_{\text {sca.j }} / \sum_{j=1}^{N_{b}} N_{j} R_{\text {sca.j }}$,

where $R_{\text {sca,j, }}$, the CPDF for scattering, is given by Eq. (34) and $N_{j}$ follows Eq. (12). Notice that our $N_{j}$ 's are in a different form compared to those shown by Lacroix et al. [16] because of $T_{\text {ref. }}$. This way of accounting scattering processes in the MC simulation also conveniently remove the requirement of a destruction/creation scheme for phonon ensembles since energy conservation is achieved statistically in the simulation. Hence, whenever an ensemble is scattered during the simulation, its frequency will be reset using Eqs. (12), (13), (14), and (38), and its direction will be re-sampled following Eqs. (19) and (22).

\subsection{Boundary conditions}

Two types of boundary condition are used here: adiabatic and isothermal boundaries. An ensemble that encounters an adiabatic boundary is reflected, which can be either specular or diffuse. In the case of specular reflection, the ensemble is simply reflected with respect to the normal vector of the boundary. For diffusive reflection, the direction cosines of the ensemble are reset randomly according to the isotropic scattering phase function, and the ensemble is re-launched without altering its energy. When an ensemble hits to an isothermal boundary, it is deleted.

\subsection{Accounting external heat generation}

Heat generation is included in our MC simulation by implementing a phonon creation scheme. It is assumed that the type of heat generation, whether Joule, laser or electron-beam heating, is not important as long as the rate of phonon production by the source is known. This requires the distribution of the volumetric power generated within the material, $\dot{g}^{\prime \prime \prime}(x, y, z)$, to be determined before the MC simulation. When $\dot{g}^{\prime \prime \prime}(x, y, z)$ is known, the amount of energy generated within each computational element and at each time step is calculated as:

$E_{\text {gen }}(x, y, z)=\dot{g}^{\prime \prime \prime} \Delta x \Delta y \Delta z \Delta t$,

and it will be added to $E(x, y, z)$ in Eq. (29) for $T_{p \text { seudo }}$ calculation. In what follows, phonon ensembles are generated continuously within the corresponding computational volume based on the $T_{p s e u d o}$ through Eqs. (11)-(14) for frequency, Eq. (15) for polarization branch, and Eqs. (19) and (22) for direction of propagation until the total energy added to the volume satisfies the amount in Eq. (39), such that:

$\sum_{i=1}^{N_{\text {engen }}} h \omega_{i} W_{\text {scaling }} \approx E_{\text {gen }}(x, y, z)$

where $N_{\text {en,gen }}$ is the number of ensembles generated for a given time step and subject to change during the simulation. 


\section{Results and discussions}

In the following sections, we first verify the $\mathrm{MC}$ simulations against the Stefan-Boltzmann law at the ballistic limit and then against the Fourier law at the diffusion limit. At the ballistic limit where phonon scattering is negligible, temperature between two isothermal boundaries follows the Stefan-Boltzmann law (SBL), which is given as:

$T^{4}=\frac{1}{2}\left(T_{L}^{4}+T_{R}^{4}\right)$

When the medium is highly scattering, heat conduction can be described accurately using the heat diffusion equation, and it is expressed as [3]:

$\nabla \cdot\left(k_{T} \nabla T\right)+\dot{\mathrm{g}}^{\prime \prime \prime}=0$,

where the thermal conductivity of silicon is given as [37]:

$k_{T}=\frac{1.585 \times 10^{5}}{T^{1.23}}$

Next the MC results with heat generation are compared against the heat diffusion equation when the medium is acoustically thick for various temperature ranges and film thicknesses. After the verifications, discussions on heat generation in silicon thin films near ballistic limit are provided. Particularly, MC results are consistently compared to the heat diffusion equation to access the validity of the diffusion approach.

\subsection{Computational parameters}

For our MC simulations, we do not specify the total number of phonon ensembles used inside the material as this quantity increases starting from the time zero and then reaches a constant value at the steady state. Since the concept of "reference temperature" is utilized, the initial temperature of the medium is always set equal to the lower temperature imposed on the isothermal boundaries. Whenever a temperature difference is applied between two boundaries, we only define the total number of ensembles emerges from the hotter boundary within a time step, $\Delta t$, and the scaling factor will be calculated and used throughout the simulation. Therefore, the number of ensembles inside our computational domain will be null initially until the first time step where ensembles are injected from the hotter boundary. In the case of an identical temperature at the isothermal boundary with heat generation, we specify the scaling factor instead of the number of ensembles at the hotter boundary. The typical number of ensembles we used to ensure that statistical fluctuations on the temperature distribution are less than $5 \%$ of the temperature difference applied is between 500 to 1000 , which are launched at each time step from the hotter boundary. The actual number of ensembles launched at each time step varies statistically depending on the total amount of incoming phonon energy at the boundary and the phonon frequency drawn. The number of division in the phonon frequency spectrum is set to be 1000 , which is sufficient to produce accurate results. We did not observe any changes to the simulation results when the frequency division was increased further to 2000 divisions. This is in line with the simulation performed by other researchers on the same topic, e.g., the work by Mazumder and Majumdar in Ref. [14].

The time step is crucial in calculating the thermal conductivity of the medium. If the chosen time step is much larger than the combined relaxation time of the medium (see Eq. (34)), then the scattering probability would always be 1 , which produces unreal- istic scattering behaviors and destroys the proper rate of heat conduction. Nevertheless, the time step should also be selected carefully such that the ballistic distance of the fastest ensemble in the domain does not exceed the smallest space step (i.e., $\Delta x$, $\Delta y$, or $\Delta z$ ) in any given time step. The current algorithm determines the time step automatically before the simulation by detecting the smallest combined relaxation time (or highest combined scattering rates) and the time for the fastest ensemble to ballistically penetrate the smallest space step, and then taking half of whichever quantity that is smaller to be the time step.

The magnitudes for the constants $B_{L}, B_{T}$, and $B_{T U}$ required in Eqs. (35)-(37) are readily available in the literature $[17,32,34,36]$. However, these constants are quite different from each other, and they depend heavily on the form of dispersion relations used in the simulation. Using the set of values provided by these works, we were not able to retrieve the correct bulk thermal conductivity since the dispersion relation in our simulation was fitted using quadratic equations, which was different from them. In addition, our treatment of scattering mechanism in the MC simulation is rather different from those in the literature $[17,32,34,36]$, but similar to the work by Lacroix et al. [16] although the authors did not explicitly list the scattering expressions and constants. Therefore, we calibrate our MC simulation based on the bulk thermal conductivity of silicon and retrieve the set of constants that correspond to our implementation, starting from those provided by Holland [36]. In Table 1, we list all the values found elsewhere $[17,32,34,36]$ and in our work for comparison purpose Figs. 1-3.

\subsection{Verification of $M C$ simulations}

Fig. 4a and b depict the comparisons between MC results and predictions by the SBL at the ballistic limit. The thickness of the film is $100 \mathrm{~nm}$. The temperature difference applied in Fig. 4a is $10-20 \mathrm{~K}$ while in Fig. $4 \mathrm{~b}$ it is $30-40 \mathrm{~K}$. It can be seen that results computed by the MC simulations match well with the SBL. For the diffusion limit verifications, we compare two cases: one with varied thermal conductivity where larger temperature difference is applied between 250 and $500 \mathrm{~K}$, and the other with constant thermal conductivity in which very small temperature difference of $4 \mathrm{~K}$ (i.e., between 298 and $302 \mathrm{~K}$ ) is imposed. A film thickness of $5 \mu \mathrm{m}$ is set for the comparison since heat conduction is diffusive based on the applied temperature differences. Fig. 4c and d show temperature distributions computed using the MC simulation and the heat diffusion equation for the two cases. Good agreement between the two sets of results is observed.

Next, MC simulations which account for heat generation inside silicon at the diffusion limit are verified against the heat diffusion equation (i.e., Eq. (42)). Temperature difference is applied between two ends of the silicon thin film while the entire film is placed under uniform heat generation. Two different film thicknesses of 5 and $6 \mu \mathrm{m}$ with applied temperature differences of $300-350 \mathrm{~K}$ and $400-450 \mathrm{~K}$ are used in the comparisons. Results are shown in Fig. 5. It is observed from Fig. $5 \mathrm{a}$ and $\mathrm{c}$ that the temperature profiles predicted by MC simulations for film thicknesses of 5 and $6 \mu \mathrm{m}$ for an applied temperature difference of 300-350 K with a heat generation strength of $2 \times 10^{15} \mathrm{~W} / \mathrm{m}^{3}$ agree well with the results computed using the heat diffusion equation. When the temperature difference is raised to $400-450 \mathrm{~K}$, we observe that MC results produce slightly higher temperature distribution than that of the heat diffusion equation (see Fig. 5c). This can be easily explained from the fact that our simulation give a slightly lower thermal conductivity at the bulk regime at $400 \mathrm{~K}$ and beyond (see Fig. 5d), which subsequently causes higher temperature distribution in the simulation. 
Table 1

Comparisons between parameters used in evaluating phonon scattering rates for silicon given in the literature and this work.

\begin{tabular}{|c|c|c|c|}
\hline \multirow[t]{2}{*}{ Source } & \multicolumn{3}{|l|}{ Parameters } \\
\hline & $\begin{array}{l}\mathrm{B}_{\mathrm{L}} \\
{\left[\mathrm{s}-\mathrm{K}^{-3}\right] ; \text { Eq. (35) }}\end{array}$ & $\begin{array}{l}\mathrm{B}_{\mathrm{T}} \\
{\left[\mathrm{K}^{-3}\right] ; \text { Eq. (36) }}\end{array}$ & $\begin{array}{l}\mathrm{B}_{\mathrm{TU}} \\
\text { [sec]; Eq. (37) }\end{array}$ \\
\hline Holland, 1963 [36] & $2.0 \times 10^{-24}$ & $9.3 \times 10^{-13}$ & $5.5 \times 10^{-18}$ \\
\hline Chen et al., 2005 [32] & $1.0 \times 10^{-30}$ & $9.3 \times 10^{-13}$ & $5.5 \times 10^{-18}$ \\
\hline Randrianalisoa and Baillis, 2008 [17] & $2.0 \times 10^{-24}$ if $\omega<\omega_{1 / 2} ; 8.03 \times 10^{-25}$ else $\omega \geqslant \omega_{1 / 2}$ & $9.3 \times 10^{-13}$ & $7.4 \times 10^{-19}$ \\
\hline Baillis and Randrianalisoa, 2009 [34] & $2.0 \times 10^{-24}$ if $\omega<\omega_{1 / 2} ; 9.4 \times 10^{-25}$ else $\omega \geqslant \omega_{1 / 2}$ & $9.3 \times 10^{-13}$ & $1.1 \times 10^{-18}$ \\
\hline Present study & $2.0 \times 10^{-24}$ & $9.3 \times 10^{-13}$ & $1.7 \times 10^{-18}$ \\
\hline
\end{tabular}

3D Schematic

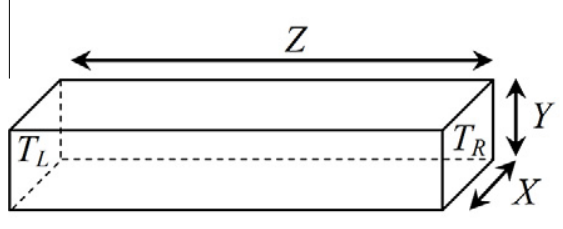

2D Top View

$\sqrt{T_{L}}$

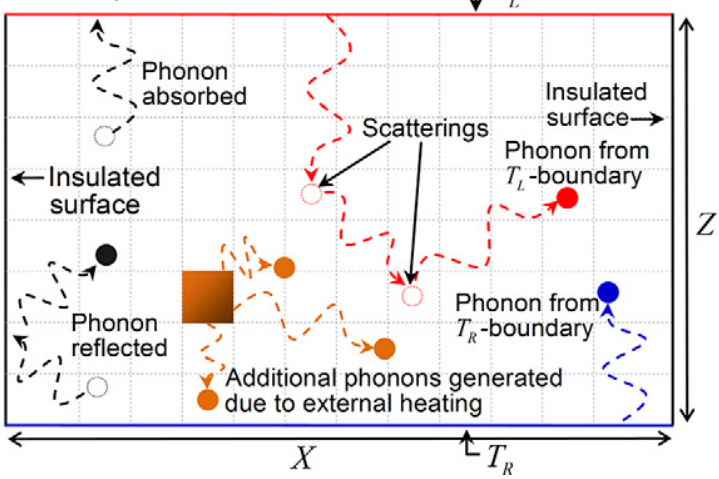

Fig. 1. The 3D-schematic and 2D-top view of the geometry considered in the Monte Carlo simulation for phonon transport.

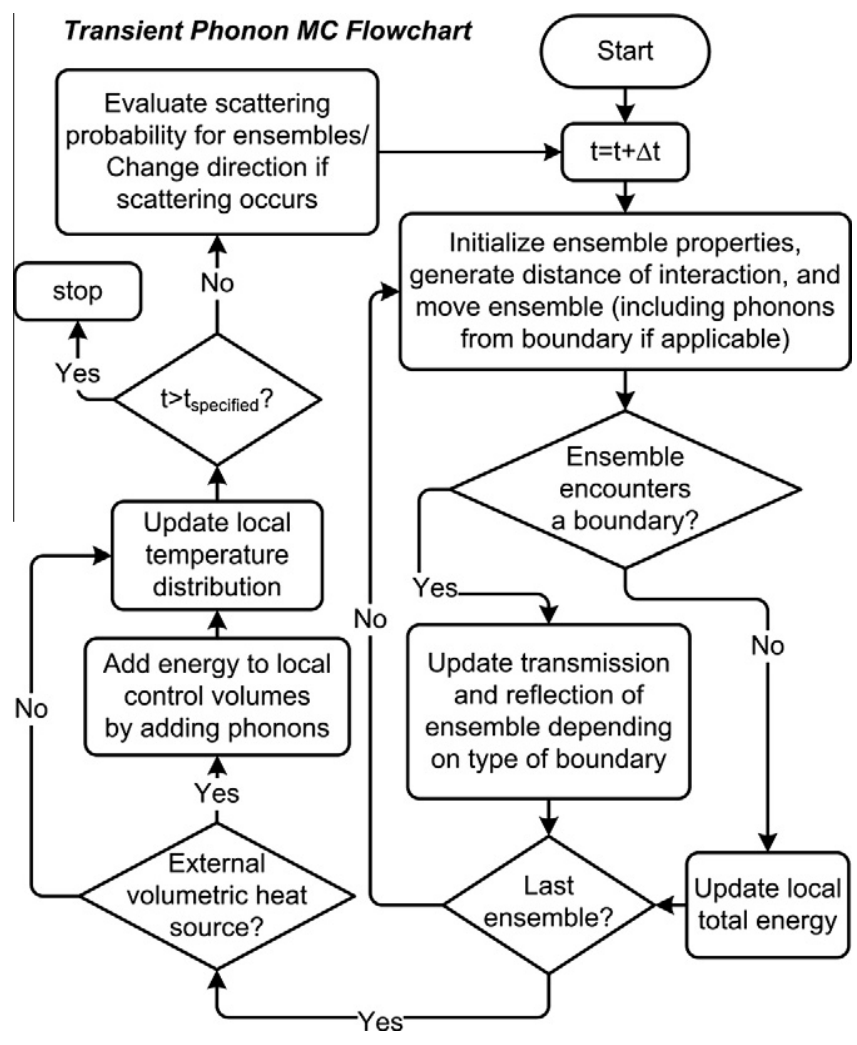

Fig. 2. The flowchart for MC phonon simulation including the volumetric heat generation.

\subsection{Size effect on thermal conductivity}

It is well-known that thermal conductivity of a material at a given temperature is reduced when the characteristic length of the

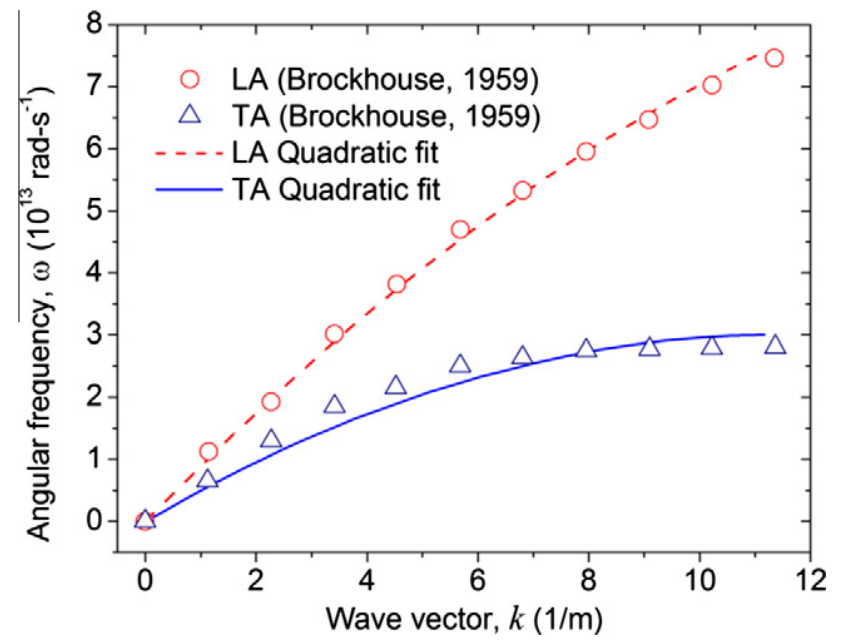

Fig. 3. Data points of the dispersion relation of silicon obtained from Brockhouse [35] and the quadratic fits of the LA-branch and TA-branch used in the MC simulation are given.

system decreases below the average mean free path of the heat carriers, owing to the ballistic heat conduction and the limiting dimension $[4,23]$. Under this condition, ballistic transport surfaces and enables heat carriers to propagate freely without scattering in a given direction. Thus the net energy exchange between two points is reduced, and hence lower thermal conductivity is observed. Using the MC simulation, the reduced thermal conductivity can be easily computed especially for nanofilms and nanowires [17,32-34,38-43]. Only the cross-plane thermal conductivities are calculated in our application. The in-plane thermal conductivity will be studied in another work. Fig. $5 \mathrm{~d}$ illustrates thermal conductivities for silicon at 300 and $400 \mathrm{~K}$ from a film thickness of $10 \mathrm{~nm}$ to $6 \mu \mathrm{m}$. Below $3 \mu \mathrm{m}$, the thermal conductivity of silicon deviates significantly from its bulk value. It is important to note 

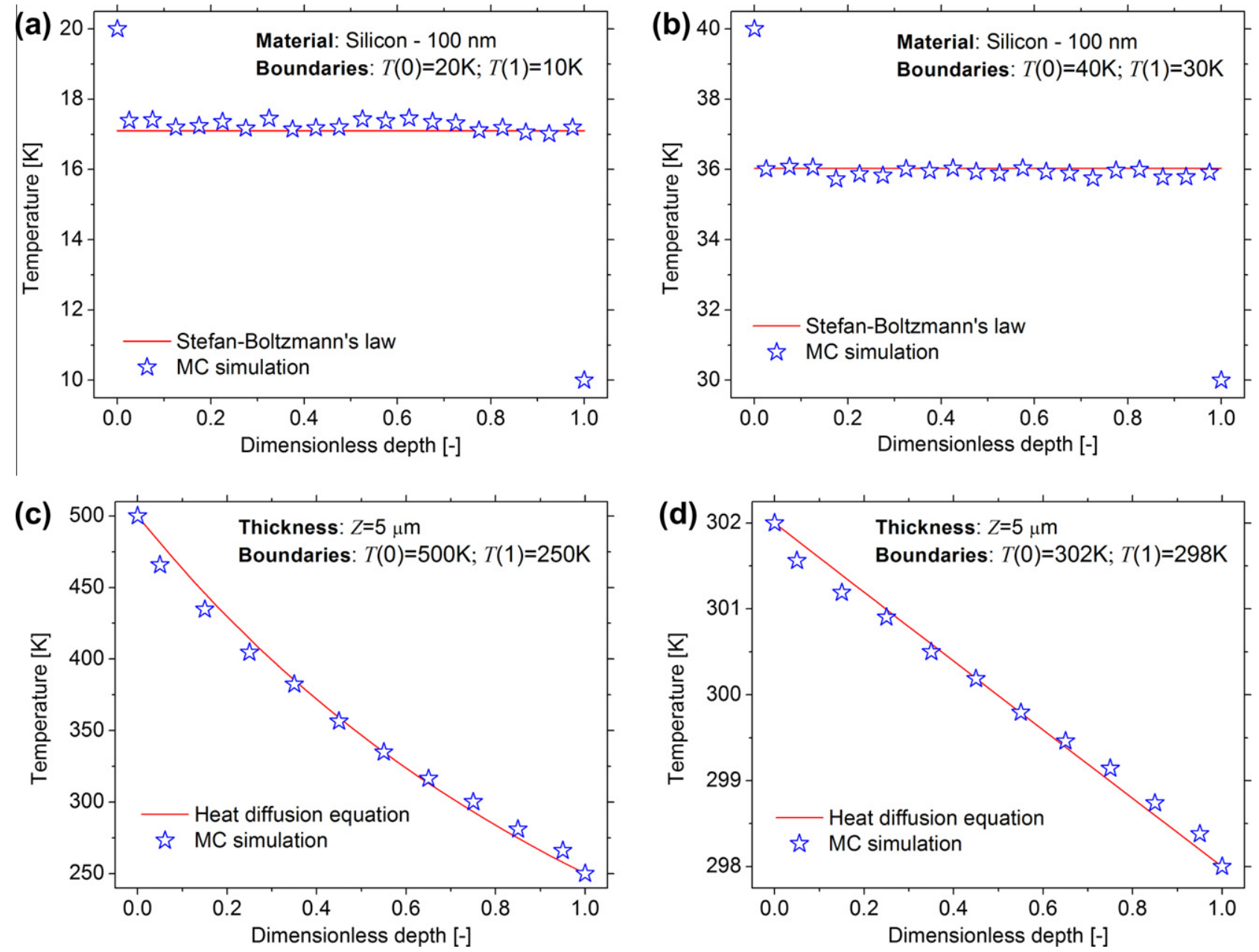

Fig. 4. Verification of MC simulation results: (a) and (b) against the Stefan-Boltzmann law at the ballistic limit and (c) and (d) against the heat diffusion equation at the diffusion limit.

that thermal conductivities are just derived values from the simulation for the sake of comparison against bulk values. Unlike the application of the Fourier law where heat flux can be easily calculated once the thermal conductivity is known assuming a linear continuous temperature profile, heat flux cannot be obtained without knowing the actual temperature distribution when ballistic transport is present. This is because discontinuities in the temperature distribution near the isothermal boundaries are evident (see Fig. 6).

\subsection{Effect of Ballistic phonon transport on thin film heating}

Using MC simulations, we studied next the effect of ballistic transport on the temperature distribution when a uniform heat source was present in the film. We compared the results against the heat diffusion equation to demonstrate the error in using the diffusion approach for solving heat conduction below the average mean free path of phonons. A temperature difference of 300$350 \mathrm{~K}$ was applied on a silicon film with thickness of 10 and $100 \mathrm{~nm}$. We used different source strengths in both cases so that the elevated temperatures are within the same range for the sake of comparison. Fig. 6a depicts the temperature distribution inside a $100 \mathrm{~nm}$ silicon film. The MC results are significantly different from those computed using the heat diffusion equation. We observe that for the same applied volumetric power generation, the diffusion method clearly underestimates the temperature distribution when compared against results from MC simulations. The volumetric power generation on the order to $10^{17}$ is not sufficient to raise temperature beyond $350 \mathrm{~K}$ while MC simulation clearly indicates that the film is heated beyond the temperatures at the boundaries. The reason for the discrepancy between diffusion approach and the MC simulation is that for a $100 \mathrm{~nm}$ silicon film the thermal conductivity is reduced from the bulk value as seen in Fig. 5d. The generated heat inside the film is conducted away slower than the bulk assumption, resulting higher temperature distribution. To have better fit against the MC results for the film, we used the thermal conductivity derived from the MC simulation at $400 \mathrm{~K}$ in the heat diffusion equation; nevertheless, we failed to set the applicability of the equation even with the modification. These results are shown by the dotted lines in Fig. 6a. Clearly, the combined effect of the reduced thermal conductivity due to the ballistic behavior and the elevated temperature renders the diffusion approach impractical even if one knows the magnitude of the reduced thermal conductivity derived from any sources. Similar observations are noted for a $10 \mathrm{~nm}$ silicon film (see Fig. 6b) with the exception that the discrepancy is more pronounced and temperature profiles are close to flat distribution as a result of intensive ballistic phonon transport.

For non-uniform heating pattern inside silicon thin film, we also observed identical behaviors as those discussed for Fig. 6a and b. The localized heating profile used here is of a Gaussian type as shown in Fig. 6c. The profile was normalized by the maximum value at the center of the film. Using different maximum source values as necessary, we tried to increase the temperature inside 

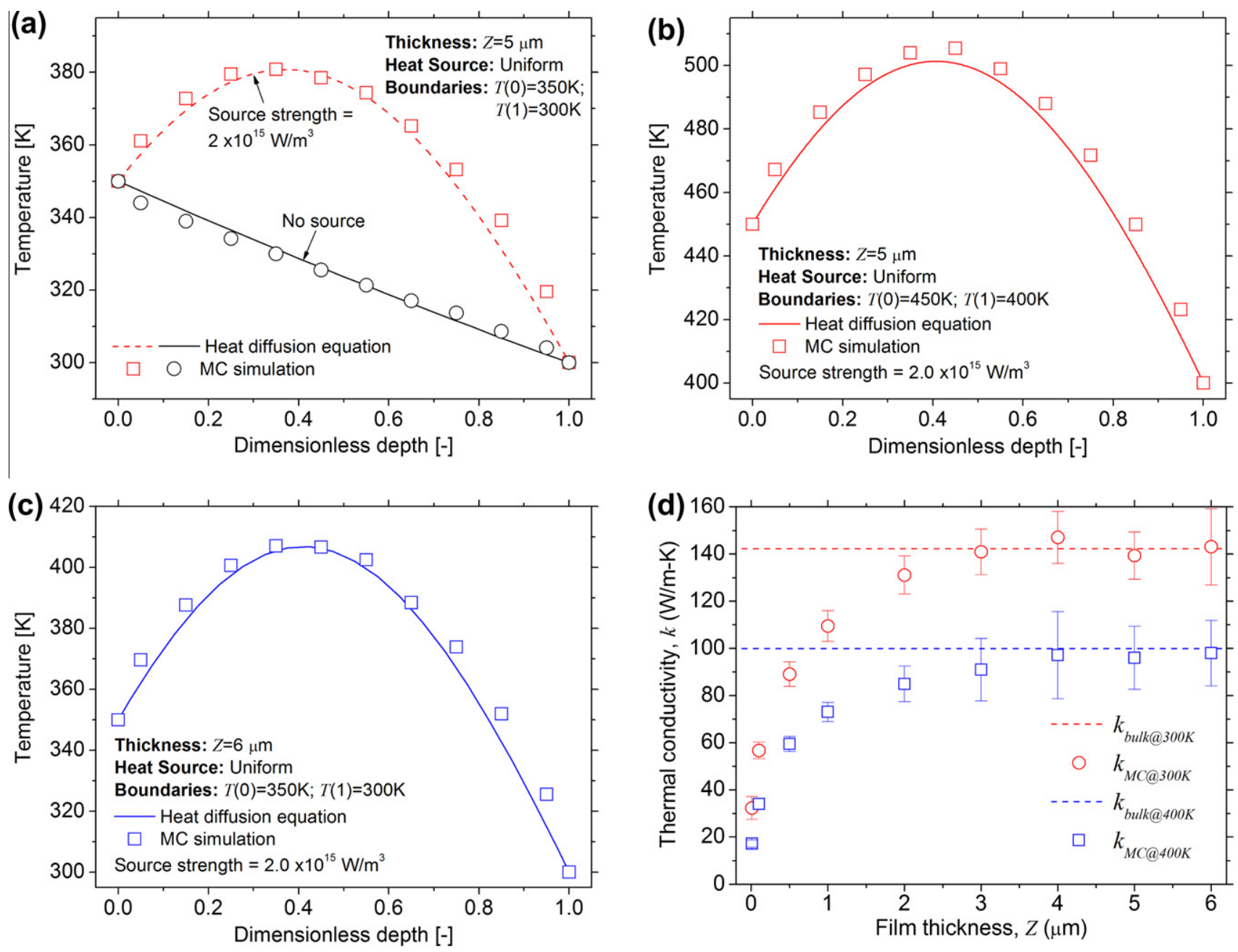

Fig. 5. (a)-(c) Verification of MC simulation results against the temperature distribution computed using the heat diffusion equation for uniformly distributed heat source. (d) The thermal conductivity and temperature distribution inside silicon thin film as a function of film thickness.

a film with thickness ranging from 10 to $5 \mu \mathrm{m}$ and with a constant temperature of $300 \mathrm{~K}$ applied at both surfaces of the film. Results are depicted in Fig. $6 \mathrm{~d}$. At the film thickness of $5 \mu \mathrm{m}$, both results from the MC simulation and diffusion approach agree well as heat conduction is diffusive. For film thicknesses of 10 and $100 \mathrm{~nm}$, the heat diffusion equation underestimates the temperature distribution inside the film. Also, discontinuities near the isothermal boundaries become increasingly pronounced when the film is reduced below $100 \mathrm{~nm}$. Results from these comparisons imply that for devices with dimension below $100 \mathrm{~nm}$, thermal conduction should not be modeled using diffusion approach as it would significantly underestimate heating and lead to device failure if not designed correctly. Although the analysis presented in this work is limited to phonon transport, similar conclusions can be expected for electron transport inside a nanodevice in which the thermal heat flux of electrons is mostly approximated using the Fourier law, even when the average mean free path of electrons is comparable to the device length.

\subsection{Temperature distribution inside a "Nanorod" due to heat generation}

The MC Results discussed thus far are for silicon thin films. In this section, we study the effect of the diffuse insulated sidewalls on the temperature distribution when heat source is present and when the geometry has finite $X$ and $Y$ (see Fig. 1). The values of $X$ and $Y$ are assumed to be 10 or $20 \mathrm{~nm}$ while the thickness $Z$ is either $100 \mathrm{~nm}$ or $500 \mathrm{~nm}$ for the purpose of comparisons. Based on the dimensions of the geometry, we termed these "nanorods." Both ends of the nanorods are set to be at $300 \mathrm{~K}$ at all times. It is then exposed to non-uniform heat generation based on the profile given in Fig. $6 c$ with a maximum of $5 \times 10^{17} \mathrm{~W} / \mathrm{m}-\mathrm{K}$ for $Z=100 \mathrm{~nm}$ and $5 \times 10^{16} \mathrm{~W} / \mathrm{m}-\mathrm{K}$ for $Z=500 \mathrm{~nm}$. Results are presented in Fig. 7a, where we notice that when the insulated sidewalls reflect diffusively, heating inside a nanorod is further increased. This is due to the fact that the diffuse reflection on the sidewalls and the small cross section $(20 \times 20 \mathrm{~nm})$ create additional scattering possibility for phonons and therefore contribute to the reduction of thermal conductivity in nanorods. For the same magnitude of heat generation along the $Z$-dimension, it is observed that thinner nanorods (with diffuse reflection on the sidewalls) cause higher temperature rise as a result of lower thermal conductivity, as evident by comparing the temperature profiles computed for the cross section of $(20 \times 20 \mathrm{~nm})$ and $(10 \times 10 \mathrm{~nm})$. It is obvious from Fig. 7a that the heat diffusion equation fails to predict any significant temperature rise in the nanorods, which is in line with observations obtained in the previous sections.

\subsection{Establishing temperature gradient inside nanostructures}

Next, we are interested in determining the order of magnitude of volumetric power generation required to increase temperature 

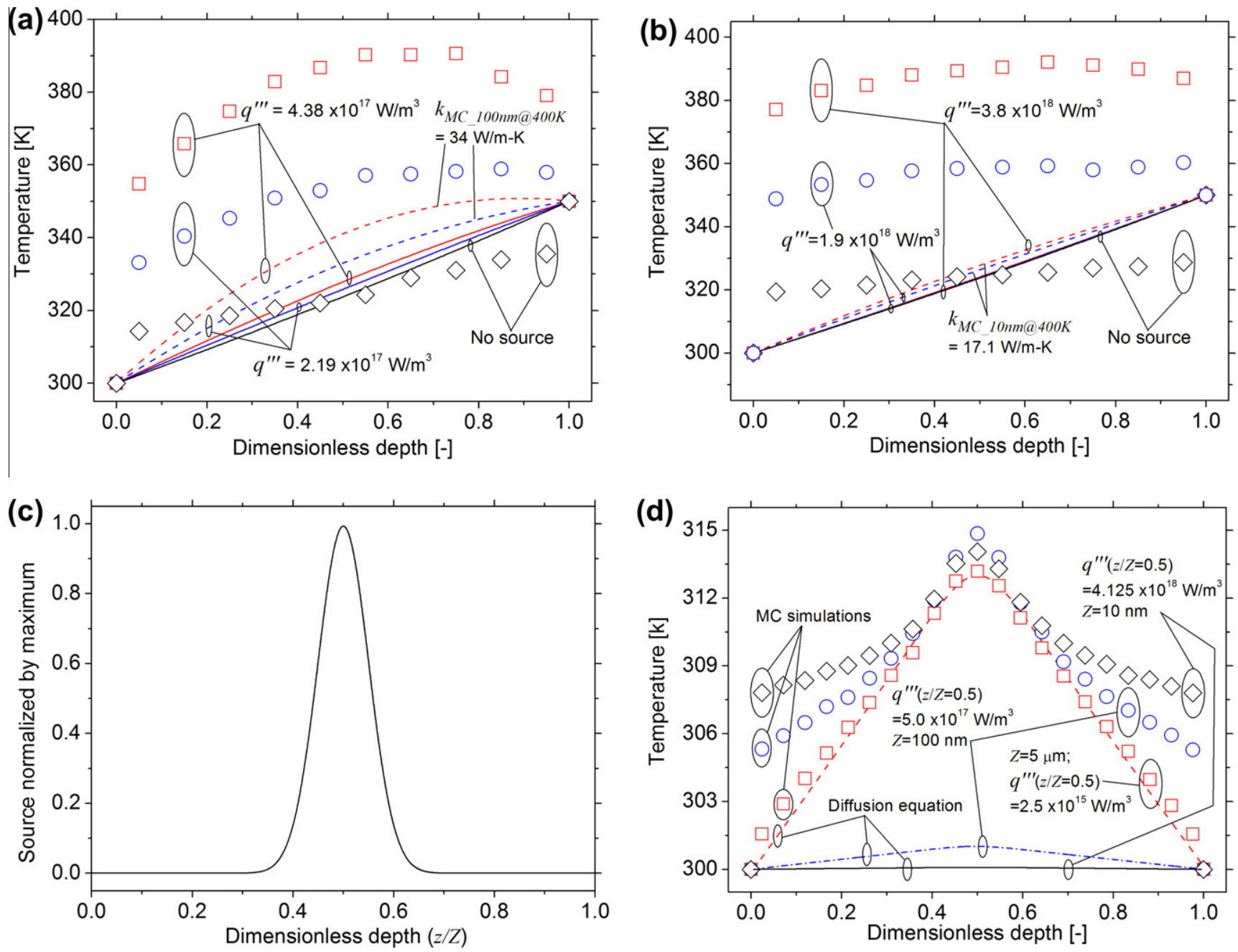

Fig. 6. (a) Temperature distributions inside silicon thin films when exposed to uniform heat sources with various strengths. Solid lines - results obtained using the heat diffusion equation, dashed lines - using the heat diffusion equation with thermal conductivity computed by MC simulation at that corresponding thickness, and symbols results computed by MC simulations. $Z=100 \mathrm{~nm}$, source is uniform in space, boundary conditions are $T(0)=300 \mathrm{~K}$ and $T(1)=350 \mathrm{~K}$. (b) Same as in (a) but with $Z=10 \mathrm{~nm}$. (c) Normalized distribution of heat source profile used in (d). (d) Temperature distribution inside silicon thin film with different thicknesses and localized heat sources.

of a nanostructure by one degree. This information is useful especially for determining if an external source is suitable for altering temperature distribution inside a nanostructure. It is already observed that the heat diffusion equation severely underestimates temperature distribution when heat source is present and therefore overestimates the required power for heating at nanoscales. Therefore, it is important to carry on MC calculations to determine the required volumetric power generation with proper physics. For this purpose we set up a thin film with one end insulated while the other is maintained at $300 \mathrm{~K}$ at all times. We then slowly increase the uniform heat source across the entire film until the temperature at the insulated end is raised by one degree. The volumetric power generation obtained this way is specific to this set of boundary conditions. For example, if both ends of the film are maintained at a constant temperature, then more power generation would be needed. Fig. 7b depicts the magnitude of the heat source required to modify temperature at the adiabatic end by one degree while the other end is kept at constant temperature. The results obtained both from the heat diffusion equation and the MC simulation are shown. Notice that the required power densities predicted by both the heat diffusion equation and the MC simulation are identical when the thickness is sufficiently large (i.e., >>3 $\mu \mathrm{m}$, see Fig. $5 \mathrm{~d}$ ). Below a film thickness of $100 \mathrm{~nm}$, however, the heat diffusion equation over-predicts the required power density by an order of magnitude or higher. To modify the temperature distribution of a silicon film with thickness below $500 \mathrm{~nm}$, it would require a heat source with strength in the order of $10^{15} \mathrm{~W} / \mathrm{m}-\mathrm{K}$ and higher, under the set of conditions described here.

It is extremely difficult to establish temperature gradient in silicon nanostructures as heat is evenly distributed due to the relatively high value of its thermal conductivity. Based on our numerous simulation trials, unless the thermal conductivity is in the order of $10^{-1} \mathrm{~W} / \mathrm{m}-\mathrm{K}$ or less (or in other words, nearly nonconductive) and if the incident power density is comparable to the those provided in Fig. 7b, the possibility of observing temperature gradient in a nanostructure is nearly null. Uniform temperature distribution assumption would be a wise choice under the prescribed conditions where heat conduction does not apply.

\subsection{Near-field thermal radiation, pulsed laser, or electron beam as heat source}

Discussions provided in the above sections are not specific to any external heating process of the nanostructures. Next we discuss the possibility of using different heating methods such as near-field thermal radiation, a pulsed laser, or an electron beam for modifying temperature distribution inside silicon thin films. Our discussions below do not target on any specific engineering 

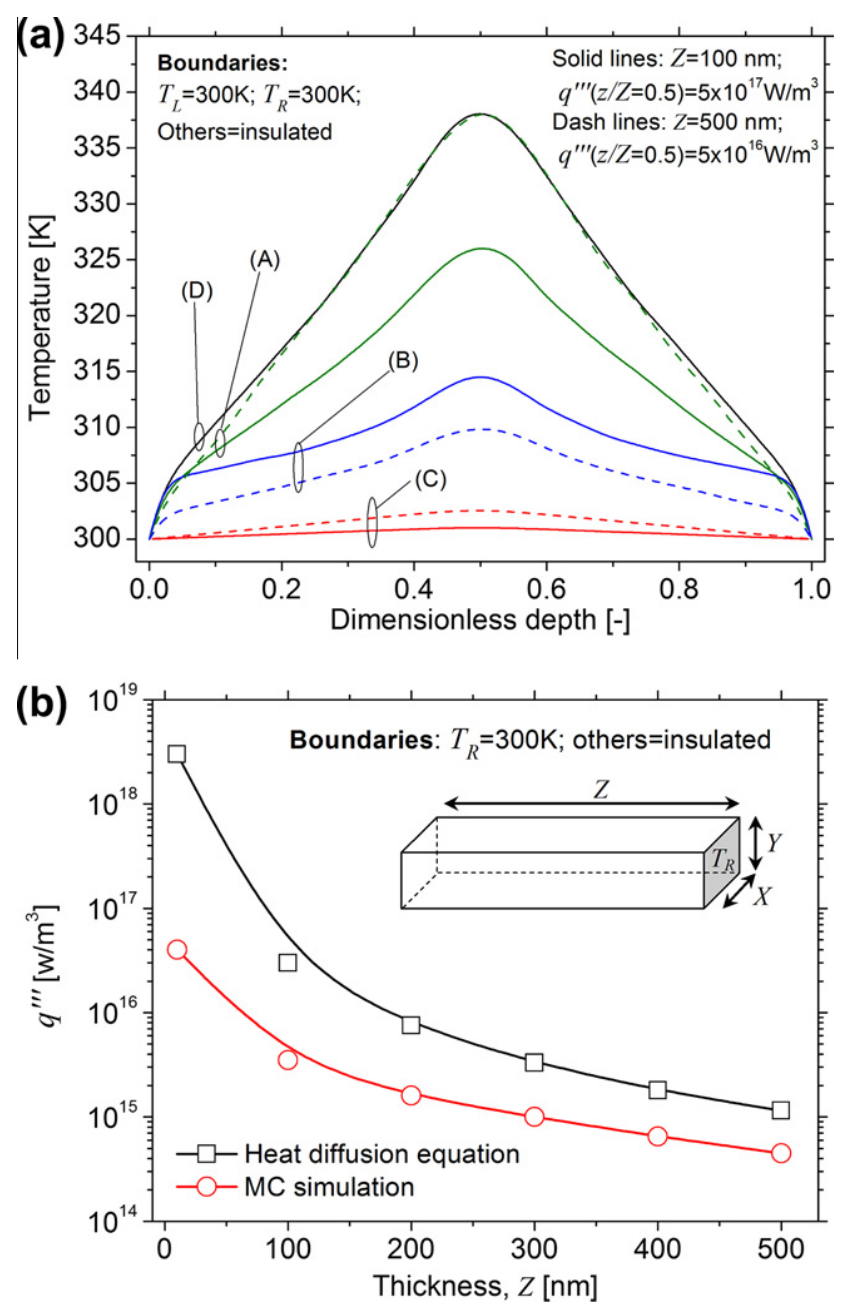

Fig. 7. (a) Temperature distributions along silicon "nanorod" with different thicknesses and localized heat sources. Case $(A)$ is where the insulated walls give diffuse reflection and $X=20 \mathrm{~nm}, Y=20 \mathrm{~nm}$, case (B) is where the insulated walls give specular reflection, case $(C)$ is the result obtained from the heat diffusion equation, and case (D) is where the insulated walls give diffuse reflection and $X=10 \mathrm{~nm}, Y=10 \mathrm{~nm}$. (b) Uniform volumetric heat generation required to increase temperature by one degree at the left (adiabatic) end.

applications, but rather we comment on the suitability of using the diffusion approach or the MC simulation on solving the problem.

\subsubsection{Near-field radiative heat transfer between a bulk and a film}

In order to estimate the magnitude of volumetric heat generation due to near-field radiative heating on a silicon thin film, near-field radiative heat exchange between a film and the bulk object needs to be solved. Near-field radiant energy exchanges between a bulk and a film, separated by a vacuum gap of thickness $d_{c}$, with perfectly smooth and parallel surfaces is schematically depicted in Fig. 8a. It is assumed that the media are in local thermodynamic equilibrium, homogeneous, isotropic, nonmagnetic, and described by a frequency-dependent dielectric function $\varepsilon_{r}(\omega)$ local in space. The system is invariant along the $\rho$-direction and azimuthally symmetric, such that only variations of the radiative flux along the $z$-direction are considered. For simplicity, the substrate on which the thin film is coated is modeled as a nonabsorbing medium with a refractive index of 1 . The bulk is maintained at constant temperature $T_{1}$, while the temperature of the film varies along the $z$-direction. The near-field radiative heat flux between the bulk and the film is calculated starting with the Maxwell equations and using the fluctuational electrodynamics
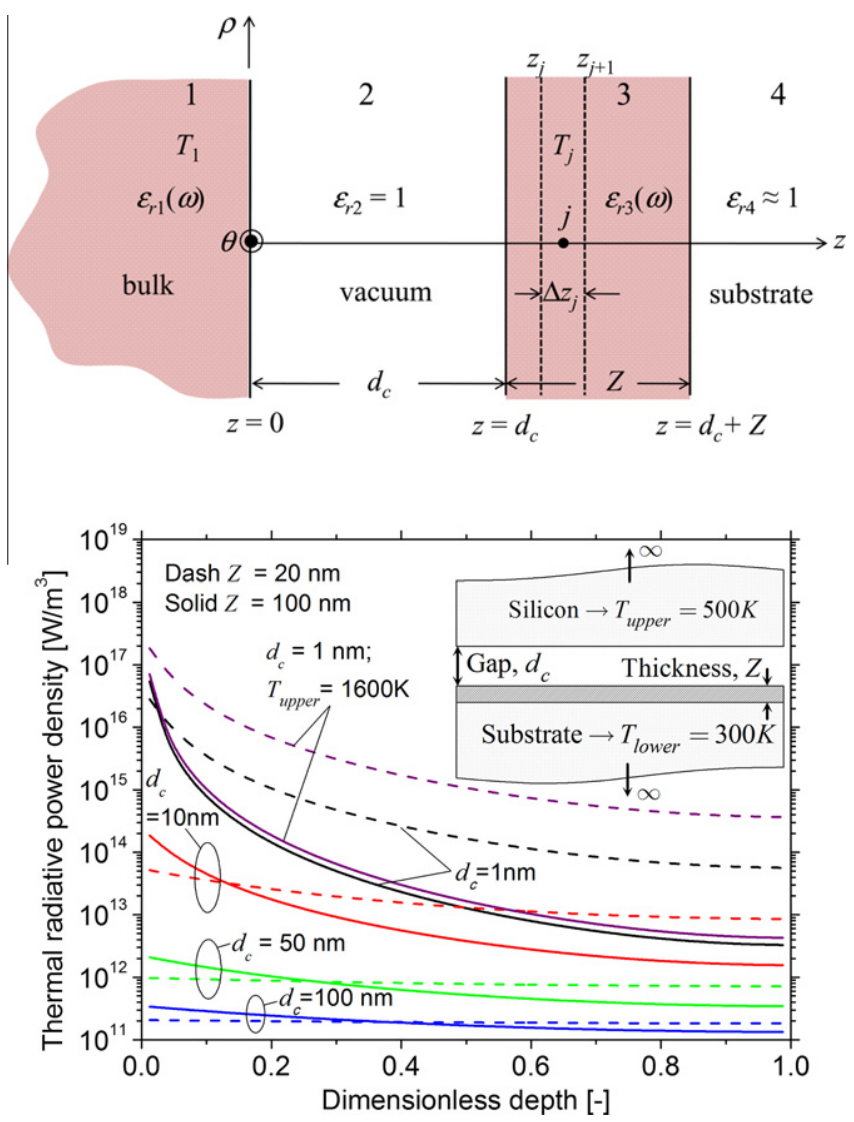

Fig. 8. (a) Schematic representation of the geometry considered: the radiative heat flux is calculated between a bulk (medium 1) and a film (medium 3 ) submerged in vacuum and separated by a gap $d_{c}$. (b) Near-field thermal radiative power density absorbed by the $n$-doped $\left(10^{17} \mathrm{~cm}^{-3}\right)$ silicon thin film as a function of gap size and thickness.

formalism, where the source of thermal radiation is modeled as a stochastic current density [44-46]. The link between the stochastic current density and the local temperature of the emitting medium is provided by the fluctuation-dissipation theorem (FDT). The monochromatic radiative flux at an arbitrary location $z_{c}$ in film 3 due to the emitting bulk 1 at temperature $T_{1}$ is determined by calculating the time-averaged $z$-component of the Poynting vector and by applying the FDT [47-49]:

$q_{\omega, 13}\left(z_{c}\right)=\frac{\omega^{2}}{\pi^{2} c_{v}^{2}} \Theta\left(\omega, T_{1}\right)$

$\operatorname{Re}\left\{i \varepsilon_{r 1}^{\prime \prime}(\omega) \int_{\beta=0}^{\infty} \beta d \beta \int_{z^{\prime}=-\infty}^{0} d z^{\prime}\left(\begin{array}{c}g_{13 \rho \alpha}^{E}\left(\beta, z_{c}, z^{\prime}, \omega\right) g_{13 \theta \alpha}^{H^{*}}\left(\beta, z_{c}, z^{\prime}, \omega\right) \\ -g_{13 \theta \alpha}^{E}\left(\beta, z_{c}, z^{\prime}, \omega\right) g_{13 \rho \alpha}^{H^{*}}\left(\beta, z_{c}, z^{\prime}, \omega\right)\end{array}\right)\right\}$

where $\beta$ is the wavevector parallel to the surfaces (i.e., the $\rho$-component of the wavevector) and $\Theta$ is the mean energy of a Planck's oscillator in thermal equilibrium. The variable $g_{13 i \alpha}^{E, H}$, where $\alpha$ involves a summation over the three orthogonal components, is the electric/magnetic plane wave representation (Weyl component) of the dyadic Green's function (DGF). The DGF can be seen as a spatial transfer function relating the fields observed at $z_{c}$ in layer 3 with frequency $\omega$ and wavevector $\beta$ to a source located at $z^{\prime}$ within medium 1. The Weyl components of the DGF are integrated in Eq. (44) over the volume of the emitter from $z^{\prime}=-\infty$ to 0 . Note that the Weyl components of the DGF needed to compute Eq. (44) have been given by Francoeur et al. [47].

The near-field radiative flux absorbed by a control volume $\Delta z_{j}$ within film 3 , delimited by the boundaries $z_{j+1}$ and $z_{j}$, is calculated 
by computing the difference between the flux crossing the boundary $z_{c}=z_{j}$ (i.e., at $z_{j}^{+}$) and the flux crossing the boundary $z_{c}=z_{j+1}$ (i.e., $z_{j+1}^{+}$). For a given separation gap $d_{c}$, the radiative heat flux absorbed by the control $\Delta z_{j}$ due to an emitting bulk at temperature $T_{1}$ is the same as the flux absorbed by medium 1 due to an emitting control volume $\Delta z_{j}$ at temperature $T_{1}$ [49]. Using this fact, the total (i.e., integrated over all angular frequencies) net near-field radiative heat flux absorbed within a control volume $\Delta z_{j}$ at temperature $T_{j}$ due to an emitting bulk maintained at temperature $T_{1}$ is given by:

$$
\begin{aligned}
& q_{\Delta z_{j}}^{a b s}=\frac{\omega^{2}}{2 \pi^{2} c_{v}^{2}} \int_{\omega=0}^{\infty} d \omega\left[\Theta\left(\omega, T_{1}\right)-\Theta\left(\omega, T_{j}\right)\right]
\end{aligned}
$$

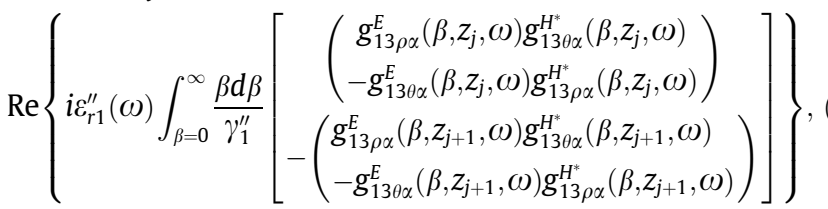

where the spatial integration over the volume of the emitter has been performed analytically [47] and $\gamma$ is the perpendicular component of the wavevector (i.e., the $z$-component of the wavevector). The heat generation term in a control volume $j$, due to near-field thermal radiation, is then calculated by dividing Eq. (45) by the volume $\Delta z_{j}$. The frequency-dependent dielectric function of doped silicon has been modeled using the formulation given by Fu and Zhang [50].

It is important to note that when using Eq. (45) to calculate the heat generation term, we do not account for the redistribution of energy inside the film due to radiant energy exchanges between the control volumes. Indeed, this contribution is negligibly small compared to heat conduction within the thin layer. Moreover, for the temperatures involved in the simulations presented hereafter, near-field thermal radiation emitted by the bulk and absorbed by a control volume $j$ dominates the value of the heat generation term, such that radiative transfer between the control volumes does not affect in a perceptible manner the heat generation term.

The near-field thermal radiative power density absorbed by the $n$-doped $\left(10^{17} \mathrm{~cm}^{-3}\right)$ silicon thin film is depicted in Fig. $8 \mathrm{~b}$ for two different film thicknesses (i.e., $Z=20$ and $100 \mathrm{~nm}$ ) as a function of various gap sizes (denoted as $d_{c}$ ) between the film and the bulk silicon. The temperature of the bulk silicon above the film is assumed to be $500 \mathrm{~K}$, which is served as heat source for the film at $300 \mathrm{~K}$. The power density absorbed within the film starts with a maximum value at the top surface and decreases exponentially along the penetration depth. It is observed that the gap size affects the power density significantly, ranging from $10^{11}-10^{17} \mathrm{~W} / \mathrm{m}^{3}$ where the gap size is decreased from 100 to $1 \mathrm{~nm}$. Under these conditions, we can ask if a temperature gradient exist. The answer lies within Fig. $7 \mathrm{~b}$ where the minimum required volumetric heat generation for creating temperature gradient is derived. For a thickness of $20 \mathrm{~nm}$, a uniform power density of $\sim 3 \times 10^{16} \mathrm{~W} / \mathrm{m}^{3}$ is needed. However, the power density absorbed by the film due to near-field thermal radiation heating is evidently below the threshold for all the gap sizes considered. Clearly, thermal conduction from the lower bulk object overwhelms the effect of near-field thermal radiative heating. On the other hand, if the bottom surface of the film is insulated rather than maintained at a constant temperature or if the film is deposited on a non-conductive substrate, near-field thermal radiative heating would elevate the film temperature, but the film would be at a uniform temperature.

The above analysis demonstrates that it is utterly difficult to establish temperature gradient inside a silicon film with a thickness of $20 \mathrm{~nm}$ unless a strong and powerful heating means is utilized. However, this may not be the case for a $100 \mathrm{~nm}$ silicon film where we observe that the minimum required power density is lowered by an order of magnitude compared to that of $20 \mathrm{~nm}$ film, as shown in Fig. 7b while the near-field thermal radiative power density does not change significantly near the top surface, provided the gap size is maintained at a few nm or less. Also, notice that if the temperature of the top bulk silicon is increased to $1600 \mathrm{~K}$ (near melting temperature of silicon at $1687 \mathrm{~K}$ ), power density absorbed by the film near the top film surface surpasses $3 \times 10^{16} \mathrm{~W} / \mathrm{m}^{3}$ for creating temperature gradient although it decreases to below $10^{15} \mathrm{~W} / \mathrm{m}^{3}$ towards the bottom film surface. Under these conditions, temperature gradient may be present in the film.

The above conclusion is drawn based on the specified set of conditions used here including a doping level of $10^{17} \mathrm{~cm}^{-3}$ for the silicon thin film for near-field enhancement in the thermal radiative exchange with the bulk silicon. For higher doping levels and distinct materials, different observations may be obtained depending on the dispersion relation and optical properties. Simulations with different set of conditions will be carried out in a future work.

\subsubsection{Pulsed laser or electron beam as heat source}

The discussions within this work mainly focus on phonon transport. For ultra-fast heating using femto-/pico-seconds pulsed lasers or electron beams, phonon transport needs to be coupled with electron transport in order to correctly describe the physics $[11,51]$. The most commonly used theory for modeling ultra-fast heating phenomena is the two-temperature model [2,9-10], which consists of two parabolic heat diffusion equations for electrons and phonons, given as:

$$
\begin{aligned}
& \text { (electrons) } \quad C_{e} \frac{\partial T_{e}}{\partial t}=-\nabla \cdot\left(k_{e} \nabla T_{e}\right)-G_{e-p h}\left(T_{e}-T_{p h}\right)+\dot{q}^{\prime \prime \prime}, \\
& \text { (phonons) } \quad C_{p h} \frac{\partial T_{p h}}{\partial t}=-\nabla \cdot\left(k_{p h} \nabla T_{p h}\right)-G_{e-p h}\left(T_{p h}-T_{e}\right) .
\end{aligned}
$$

Through initial intensive heating by the pulsed laser, electrons gain energetic energy from photons and therefore the electron temperature increases while phonons remain at their initial temperature. It is then by electron-phonon scattering, the phonon temperature is elevated. In this situation, phonons do not directly interact with the source, which necessitates the coupling between electron and phonon transport. When average phonon mean free path is larger than the object dimension, Eq. (47) needs to be replaced with the BTE, which can be solved using the MC simulation. Same applies for Eq. (46). However, if the heating power density distribution is insufficient to establish temperature gradient, then it is not necessary to apply the MC simulation assuming that all the boundaries are insulated. In other words, the term $-G_{e-p h}\left(T_{p h}-T_{e}\right)$ in Eq. (47) needs to be highly non-uniform in space such that:

$\frac{G_{e-p h} Z^{2}}{k_{M C, p h}}>>1$,

to ensure that temperature gradient exists. Nevertheless, if isothermal boundaries are involved in the analysis, the MC simulation is always required especially when the mean free path is comparable to the object dimension.

\section{Conclusions}

In this study, nanoscale phonon transport within silicon structures with different aspect ratios subjected to internal heat generation was explored. A new MC simulation algorithm was developed for phonon transport, which was different from those available in the literature in the way that a "reference temperature" was used to eliminate unnecessary additional ensemble tracings. The "reference temperature" was set identical to the initial 
temperature and the transient temperature never falls below this value. Only phonons in addition to those presented at the "reference temperature" were simulated to reduce the memory storage and computational resources. Because of this implementation in the MC simulation, additional modifications to some of the steps for phonon initialization, scattering, and pseudo-temperature calculation were required and presented accordingly. Propagations of phonons in the transverse and longitudinal polarization branches were also accounted for in the calculations. MC Results were verified against the Stefan-Boltzmann law at the ballistic limit and the heat diffusion equation at the diffusion limit. In addition, phonon transport due to heat generation for various film thicknesses was also studied and compared against the heat diffusion equation. It was found that the heat diffusion equation significantly underestimates temperature distribution at nanoscales in the presence of external heat source due to the reduced thermal conductivity. Using the reduced thermal conductivity computed by the MC simulation in the heat diffusion equation, the resultant temperature distribution was still incorrect compared to that produced by the MC simulation. This implies that the heat diffusion equation is not suitable at all for calculating temperature distribution in the presence of heat source at nanoscales, even if the correct reduced thermal conductivity is known. This is important because temperature increase in a nanodevice subjected to heat generation is much higher than those predicted using diffusion approaches, and can lead to device failure if not designed properly.

A simple analysis of using different possible heat sources, including near-field radiative heat transfer, pulsed-laser beam, and electron-beam for heating silicon thin films was also provided. To ensure that a temperature gradient exists in nanodevices especially ones with dimensions smaller than the mean free path of phonons, a powerful source in the order of $10^{15} \mathrm{~W} / \mathrm{m}^{3}$ and above (for device length less than $200 \mathrm{~nm}$ ) is required, given the set of parameters applied here. Otherwise, uniform phonon-temperature assumption is acceptable for thermal analysis.

\section{Acknowledgments}

This work was initiated and sponsored by the Kentucky Science and Engineering Foundation (Grant No: KSEF-1147-RDE-009 and Grant No: KSEF-1718-RDE-011) when all authors were at the University of Kentucky in Lexington, KY, USA. The subsequent effort and completion of this work were carried out by Basil T. Wong at Swinburne University of Technology, Sarawak Campus, Malaysia where he was appointed Senior Lecturer. Partial support for M. P. Mengüç was received from the FP-7-PEOPLE-IRG-2008 (Grant No: 239382) at Özyeğin University, Istanbul, Turkey.

\section{References}

[1] N.W. Ashcroft, N.D. Mermin, Solid State Physics, Saunders Company, Philadelphia, 1976.

[2] G. Chen, Nanoscale Energy Transport and Conversion, Oxford Press, New York, 2005.

[3] F.P. Incropera, D.P. DeWitt, Fundamentals of Heat and Mass Transfer, 4th ed., John Wiley \& Sons, New York, 1996.

[4] J.M. Ziman, Principles of the Theory of Solids, Cambridge University Press, Cambridge, 1964.

[5] A. Aste, R. Vahldieck, M. Rohner, Full hydrodynamic simulation of GaAs MESFET's, Int. J. Numerical Model. Electron. Netw. Device. Field. 17 (2004) 4359.

[6] J. Lai, A. Majumdar, Concurrent thermal and electrical modeling of submicrometer silicon devices, J. Appl. Phys. 79 (9) (1996) 7353-7361.

[7] A. Majumdar, in: C.L. Tien (Ed.), Microscale Energy Transport, Begell House, New York, 1998, pp. 1-93.

[8] K. Tomizawa, Numerical Simulation of Submicron Semiconductor Devices, Artech House, Inc., Norwood, 1993.

[9] B.T. Wong, M.P. Mengüç, Nanoscale Thermal Transport for Applications in Micro/Nanomachining, Springer, 2008.

[10] D.Y. Tzou, Macro- to Microscale Heat Transfer, Taylor \& Francis, Washington, DC, 1997.
[11] B.T. Wong, M.P. Mengüç, R.R. Vallance, Nanoscale thermal transport induced by electron-beam, Int. J. Heat Mass Transfer 50 (2007) 5099-5107.

[12] B.T. Wong, M.P. Mengüç, Analysis of electrical and thermal responses of ndoped silicon to an impinging electron beam and Joule heating, Int. J. Heat Mass Transfer 52 (11-12) (2009) 2632-2645.

[13] A.A. Joshi, A. Majumdar, Transient ballistic and diffusive phonon heat transport in thin films, J. Appl. Phys. 74 (1) (1993) 31-39.

[14] S. Mazumder, A. Majumdar, Monte Carlo study of phonon transport in solid thin films including dispersion and polarization, J. Heat Transfer 123 (2001) 749-759.

[15] G. Chen, Ballistic-diffusive equations for transient heat conduction from nano to macroscales, J. Heat Transfer 124 (2002) 320-328.

[16] D. Lacroix, K. Joulain, D. Lemonnier, Monte Carlo transient phonon transport in silicon and germanium at nanoscales, Phys. Rev. B 72 (2005) 064305.

[17] J. Randrianalisoa, D. Baillis, Monte Carlo simulation of steady-state microscale phonon heat transport, J. Heat Transfer 130 (2008) 072404.

[18] L.-C. Liu, M.-J. Huang, Thermal conductivity modeling of micro- and nanoporous silicon, Int. J. Therm. Sci. (2010), (in press)

[19] Z. Wang, R. Zhao, Y. Chen, Monte Carlo simulation of phonon transport in variable cross-section nanowires, Sci. China Technol. Sci. 53 (2) (2010) 429434.

[20] M.-J. Huang, T.-C. Tsai, L.-C. Liu, A Study of Phonon Transport in Si/Ge Superlattice Thin Films Using a Fast MC Solver, Journal of Electronic Materials (2010) DOI: 10.1007/s11664-009-1066-y.

[21] C. Cercignani, The Boltzmann Equation and Its Applications, vol. 67, SpringerVerlag New York, Inc., New York, 1988.

[22] B.T. Wong, M.P. Mengüç, Monte Carlo methods in radiative transfer and electron-beam processing, J. Quant. Spectrosc. Radiat. Transfer 84 (2004) 437450.

[23] J.M. Ziman, Electrons and Phonons, Oxford University Press, London, 1960.

[24] S.-Y. Li, H.-S. Chu, W.-M. Yan, Numerical study of phonon radiative transfer in porous nanostructures, Int. J. Heat Mass Transfer 51 (2008) 3924-3931.

[25] D. Lacroix, I. Traore, S. Fumeron, G. Jeandel, Phonon transport in silicon, influence of the dispersion properties choice on the description of the anharmonic resistive mechanisms, The Eur. Phys. J. B 67 (2009) 15-25.

[26] D. Lemonnier, in: Microscale and Nanoscale Heat Transfer, in: S. Volz (Ed.), Topics Appl. Physics, vol. 107, Springer, Berlin, Heidelberg, 2007, pp. 77106.

[27] J. Baxter, Z. Bian, G. Chen, D. Danielson, M.S. Dresselhaus, A.G. Fedorov, T.S. Fisher, C.W. Jones, E. Maginn, U. Kortshagen, A. Manthiram, A. Nozik, D.R. Rolison, T. Sands, L. Shi, D. Shollh, Y. Wu, Nanoscale design to enable the revolution in renewable energy, Energy Environ. Sci. 2 (2009) 559-588.

[28] Z.M. Zhang, Nano/Microscale Heat Transfer, McGraw-Hill, New York, 2007.

[29] O. Essner, P. Dollfus, S. Galdin-Retailleau, J. Saint-Martin, Improved Monte Carlo algorithm of phonon transport in semiconductor nanodevices, J. Phys. Conf. Series 92 (2009) 012079.

[30] Q. Hao, G. Chen, M.-S. Jeng, Frequency-dependent Monte Carlo simulations of phonon transport in twodimensional porous silicon with aligned pores, J. Appl. Phys. 106 (2009) 114321.

[31] M.-S. Jeng, R. Yang, D. Song, G. Chen, Modeling the thermal conductivity and phonon transport in nanoparticle composites using Monte Carlo simulation, J. Heat Transfer 130 (2008) 042410-042411.

[32] Y. Chen, D. Li, J.R. Lukes, A. Majumdar, Monte Carlo simulation of silicon nanowire thermal conductivity, J. Heat Transfer 127 (2005) 1129-1137.

[33] D. Lacroix, K. Joulain, D. Terris, D. Lemonnier, Monte Carlo modeling of phonon transport in nanodevices, J. Phys. Conf. Series 92 (2007) 012078.

[34] D. Baillis, J. Randrianalisoa, Prediction of thermal conductivity of nanostructures: influence of phonon dispersion approximation, Int. J. Heat Mass Transfer 52 (2009) 2516-2527.

[35] B.N. Brockhouse, Lattice vibration in silicon and germanium, Phys. Rev. Lett. 2 (6) (1959) 256-258.

[36] M.G. Holland, Analysis of lattive thermal conductivity, Phys. Rev. 132 (6) (1963) 2461-2471.

[37] R.F. Wood, G.E. Giles, Macroscopi theory of pulse-laser annealing, I. thermal transport and melting, Phys. Rev. B 23 (6) (1981) 2923-2942.

[38] W. Liu, M. Asheghi, Phonon-boundary scattering in ultrathin single-crystal silicon layers, Appl. Phys. Lett. 84 (19) (2004) 3819-3821.

[39] Y. Ju, K.E. Goodson, Phonon scattering in silicon films with thickness of order $100 \mathrm{~nm}$, Appl. Phys. Lett. 74 (20) (1999) 3005-3007.

[40] M. Asheghi, M. Toulzebaev, K.E. Goodson, Y. Leung, S. Wong, Temperature dependent thermal conductivity of single-crystal silicon layers in SOI substrates, J. Heat Transfer 120 (1) (1998) 30-36.

[41] W. Liu, M. Asheghi, Thermal conduction in ultrathin pure and doped single crystal silicon layers at high temperatures, J. Appl. Phys. 98 (12) (2005) 123523.

[42] W. Liu, M. Asheghi, Thermal conductivity measurements of ultra-thin single crystal silicon layers, J. Heat Transfer 128 (1) (2006) 75-83.

[43] D. Terris, K. Joulain, D. Lemonnier, D. Lacroix, P. Chantrenne, Prediction of the thermal conductivity anisotropy of Si nanofilms - Results of several numerical methods, Int. J. Therm. Sci. 48 (2009) 1467-1476.

[44] S.M. Rytov, Y.A. Kravtsov, V.I. Tatarskii, Principles of Statistical Radiophysics 3: Elements of Random Fields, Springer, Berlin, Heidelberg, New York, 1989.

[45] M. Francoeur, M.P. Mengüç, Role of fluctuational electrodynamics in near-field radiative heat transfer, J. Quant. Spectrosc. Radiat. Transfer 109 (2008) 280293. 
[46] M. Francoeur, M.P. Mengüç, R. Vaillon, Local density of electromagnetic states within a nanometric gap formed between thin films supporting surface phonon polaritons, J. Appl. Phys. 107 (2010) 034313.

[47] M. Francoeur, M.P. Mengüç, R. Vaillon, Solution of near-field thermal radiation in one-dimensional layered media using dyadic Green's functions and the scattering matrix method, J. Quant. Spectrosc. Radiat. Transfer 110 (2009) 2002-2018.

[48] M. Francoeur, M.P. Mengüç, R. Vaillon, Near-field radiative heat transfer enhancement via surface phonon polaritons coupling in thin films, Appl. Phys. Lett. 93 (2008) 043109.
[49] M. Francoeur, M.P. Mengüç, R. Vaillon, Spectral tuning of near-field radiative heat flux between two thin silicon carbide films, J. Phys. D Appl. Phys. 43 (2010) 075501.

[50] C.J. Fu, Z.M. Zhang, Nanoscale radiation heat transfer for silicon at different doping levels, Int. J. Heat Mass Transfer 49 (2006) 1703-1718.

[51] D.Y. Tzou, K.S. Chiu, Temperature Dependent Thermal Lagging in Ultra Fast Laser Heating, International Journal of Heat and Mass Transfer 44 (2001) 1725-1734. 